Original article

\title{
Using pharmacopuncture for chronic tension-type headache treatment
}

\author{
Lyudmila N. Belimova1, Viktor A. Balyazin ${ }^{1}$ \\ ${ }^{1}$ Rostov State Medical University, Rostov, Russia \\ Received 3o June 2018, Accepted 26 May 2021
}

Original Text (C) Lyudmila N. Belimova, Viktor A. Balyazin, 2018, published in Saratov Journal of Medical Scientific Research 2018; 14 (3): $380-384$.

(C) 2021, Belimova L. N., Balyazin V.A.

(C) 2021, Saratov Medical Journal

\begin{abstract}
:
Objective: assessing pharmacopuncture effect on pain syndrome and level of anxiety-depressive disorders in patients with chronic tension-type headache (CTTH).

Materials and methods. An open-label controlled trial was conducted on 95 CTTH patients split among two groups by means of adaptive randomization. The comparison group subjects were receiving $35-70 \mathrm{mg} /$ day of amitriptyline and $450 \mathrm{mg} / \mathrm{day}$ of tolperisone, while patients of the treatment group were assigned 2-4 courses of pharmacopuncture by the original method. The results were evaluated on the basis of the scores obtained via Visual Analogue Scale (VAS), McGill Pain Questionnaire, Beck Depression Inventory (BDI), and State-Trait Anxiety Inventory (STAI) sensu Ch. Spielberger and Yu. Khanin.

Results. Patients in both clinical groups were inclined to a positive evaluation of received treatment. Based on the results of McGill Pain Questionnaire, we determined both affective and sensory pain descriptors, with a predominance of the latter. Both groups exhibited moderate depressive symptoms according to BDI of the psychological status. Prevalence of trait anxiety over reactive anxiety was determined by STAI. According to VAS, pain intensity after the treatment declined from $5.26 \pm 0.87$ to $1.7 \pm 0.78$ points $(\mathrm{p}<0.05)$ and from $5.17 \pm 0.78$ to $1.65 \pm 0.79(\mathrm{p}<0.05)$ in the comparison group versus the treatment group. An improvement of anxiety and depression indicators was detected.

Conclusion. Our results have demonstrated that pharmacopuncture is very promising in treating CTTH patients.
\end{abstract}

Keywords: chronic tension-type headache, non-pharmacological treatment, reflexology, pharmacopuncture.

Cite as Belimova LN, Balyazin VA. Using pharmacopuncture for chronic tension-type headache treatment. Saratov Medical Journal 2021; 2(2): e0201

Correspondence to Lyudmila N. Belimova, Tel.: +7(989)6164260. E-mail: $\underline{\text { belimova-ln@yandex.ru }}$

\section{Introduction}

In current medical information systems, it is possible to find many publications dedicated to the problem of chronic tension-type headache (CTTH). The attention of domestic and foreign authors could be explained by high prevalence of this pathology (up to $4 \%$ of the population; $65 \%$ of affected individuals are women [1]), imperfection of existing methods of CTTH diagnosis and treatment, as well as by the search for new ways to solve this problem.

In 2010, Bendtsen L. et al. [2] published a directive of the European Federation of Neurological Societies (EFNS) on the diagnosis and treatment of tension-type headache (TTH). Six years later, similar domestic clinical guidelines emerged, which are subject to revision every three years [3]. Up to date, no specific diagnostic markers have been identified for CTTH, and the diagnosis is still based on the criteria of the International Classification of Headache Disorders (ICHD) of the second and third revisions, which, by the way, did not changed in relation to TTH. Treatment recommendations are based on the efficacy evidence of certain CTTH treatment procedures, as well as on the presence of contraindications to their use, and on a standpoint of decreasing the medicamentous drug load.
On the basis of the contemporary CTTH pathogenesis concept, various medications are employed in everyday practice - such as simple analgesics, nonsteroidal antiinflammatory drugs (NSAIDs), antidepressants, central muscle relaxants, as well as their combinations. The use of tricyclic antidepressants (e.g., amitriptyline) in CTTH patients has A recommendation level (1a level of evidence), which is due to its central analgesic effect, as well as a direct influence on anxiety-depressive disorders that are often inherent in CTTH patients. The drug load in such patients is accompanied by a variety of undesirable side effects that can be reduced or eliminated by using non-pharmacological approaches to treating the CTTH.

The use of acupuncture in CTTH patients, in accordance with available recommendations, has $\mathrm{C}$ recommendation level, i.e., suggestions were made in the absence of good quality clinical trials (level of evidence 2b/3) [2, 3]. In addition to the lack of multicenter randomized controlled trials, a number of factors, overlooked by the authors, affected the level of evidence for reflexology use in CTTH. The effectiveness of reflexology methods for various pain syndromes directly depends on the technique of needle placement, frequency and duration of sessions, the approach to determining the impact points, as well as on the specific 
method choice per se (acupuncture, pharmacopuncture, electrical stimulation, heating, massage, etc.).

Despite this fact, an updated Cochrane Review on the acupuncture use in preventive therapy of CTTH (12 studies; 2,349 participants) demonstrated the efficacy of acupuncture in CTTH treatment, even though the authors insisted on further research, especially on comparing the acupuncture with other treatment options [4]. The authors of another review, involving 10 randomized controlled trials of patients with TTH, emphasized the superiority of reflexology over its imitation, thereby considering it more effective than medicamentous therapy [5].

Recently, a group of Chinese scholars published the results of their project on studying the biomarkers of depression and anxiety, monitoring their severity, and on antidepressant effects of acupuncture. It was shown that in experimental models of depression in rats, the content of brain-derived neurotrophic factor (BDNF) and its messenger RNA in blood serum and hippocampus was significantly less than in the control group, and the use of acupuncture has positively changed this picture [6].

An annual increase in the number of published randomized controlled trials, systematic reviews and metaanalyzes demonstrates their clinical effectiveness rather than solely the viability of reflexology analgesia methods [7]. The noteworthy adherence of patients with chronic headaches to non-pharmacological treatment methods [8], as well as encouraging results of the reflexology therapy use, make further studies in this field promising.

Objective: to assess an effect of pharmacopuncture on pain syndrome and level of anxiety-depressive disorders in patients with chronic tension-type headache.

\section{Materials and methods}

An open-label, controlled trial enrolled 95 patients with CTTH aged 19 to 60 years old (male to female ratio 1:5). The study was carried out in agreement with the independent local ethics committee (Minutes of the Ethics Committee Meeting No. 15/13 of 24 October, 2013) at the neurology department of the City Hospital No. 6 of Rostov-on-Don and at Pskov City Polyclinic in the period from 2014 to 2017. The selection of patients was carried out on the basis of the established diagnosis of CTTH compliance with the ICHD diagnostic criteria of the third revision [9], as well as in the absence of various organic brain diseases, along with severe somatic, neurological and psychiatric pathologies.

The eligible patients were randomly assigned to two clinical groups (comparison group and treatment group) after reviewing the information sheet and completing the informed consent form. The randomization of the distribution among groups was provided by the adaptive randomization technology, thereby maintaining the possibility of sustaining an equal number of patients in the samples during the entire randomization procedure. The resulting groups were comparable in terms of clinical and demographic indicators (Table).

Patients in the comparison group were receiving medicamentous therapy sensu clinical guidelines, consisting of taking tricyclic antidepressants $(30-75 \mathrm{mg} /$ day of amitriptyline) and central muscle relaxants (450 $\mathrm{mg} /$ day of tolperisone). The dosage of preparations was selected individually, taking into account their tolerability and recommendations for their use.
Table. Clinical groups of CTTH patients: general traits

\begin{tabular}{|l|c|c|}
\hline \multicolumn{1}{|c|}{ Parameter } & $\begin{array}{c}\text { Comparison group } \\
(\mathrm{n}=47)\end{array}$ & $\begin{array}{c}\text { Treatment group } \\
(\mathrm{n}=48)\end{array}$ \\
\hline Gender, male/female & $7 / 40$ & $9 / 39$ \\
\hline Mean age, years & $36.5 \pm 9.1$ & $35.5 \pm 8.8$ \\
\hline $\begin{array}{l}\text { CTTH duration, } \\
\text { years }\end{array}$ & $4.5 \pm 1.7$ & $4.2 \pm 1.8$ \\
\hline
\end{tabular}

The treatment group patients received therapy in the form of 2-4 courses of pharmacopuncture 7 days apart, according to the original method (patent application for invention No. 2018107387 of 27 February, 2018). Each pharmacopuncture course consisted of three procedures of administering $0.5-1 \mathrm{ml}$ of a $0.5 \%$ procaine solution into paired and unpaired acupuncture points of the head and neck (19TE, 20TE, 2O(19)GB, 14(13)GB, EX-HN3 sensu international nomenclature [10]) with a frequency of every 4 days. When several episodes of headache occurred, patients of both clinical groups were taking $400 \mathrm{mg}$ of ibuprofen.

All patients underwent a standard clinical and neurological examination. To confirm the diagnosis and evaluate the subsequent therapy, a headache diary was kept four weeks before and throughout the treatment. The headache intensity and qualitative assessment of its nature were determined using the visual analogue scale (VAS) and the McGill Pain Questionnaire. The presence of anxietydepressive disorders was assessed using State-Trait Anxiety Inventory (STAI) sensu Spielberger-Khanin scale of personal and situational anxiety, and Beck Depression Inventory (BDI). To evaluate the treatment outcomes, repeated testing was conducted two months after the onset of therapy.

The data were processed using the SPSS 10.0 software package. Means (M) and standard deviations (SD) were determined. Data are presented as $\mathrm{M} \pm \mathrm{SD}$. The normality of distribution in the samples was confirmed using the Kolmogorov-Smirnov test. Given the small sample sizes, the nonparametric Wilcoxon-Mann-Whitney test was used to assess the differences between the groups. Evaluation of the treatment effectiveness was carried out using the Wilcoxon test for related samples. Differences were considered statistically significant at $\mathrm{p}<0.05$.

\section{Results}

Examination of CTTH patients did not reveal neither somatic disorders, nor focal neurologic signs. The nature of the patient complaints was conventional and did not differ from the ICHD- 3 criteria. The anamneses were typical and did not contain any physical or mental abnormalities in the past. The duration of CTTH in the groups averaged approximately four years. All patients responded positively to the therapy, noting an improvement in their general condition, a reduction in the headache intensity and its frequency of occurrence. In the patients of the treatment group, the visible effect of the treatment was appearing somewhat earlier than in the comparison group, which was expressed in the improvement of the emotional background and mood, and positive subjective assessment of the treatment.

The intensity of headache seizures according to the VAS scale in both groups was assessed as moderate, less often as severe, and did not differ significantly among the groups. As a result of conducted therapy in both groups, there was a significant reduction $(\mathrm{p}<0.05)$ in headache intensity 
indicators on the VAS scale (Figure 1). At the time of testing, the patients rated the headache attack intensity as mild, and on seven occasions they declared the complete absence of pain.

The average values of the evaluative scale of the McGill Pain Questionnaire (a variant of the verbal rank scale) before the treatment onset were: $2.5 \pm 0.5$ points in the comparison group and $2.5 \pm 0.6$ points in the treatment group. Thus, the patients' subjective assessment of the headache intensity was mainly expressed as severe.

In both groups of patients, McGill Pain Index (MGPI) was augmented mainly due to the indicators of the sensory scale (Figure 2). The total number of selected pain descriptors (NSPD) in clinical groups did not differ significantly as well

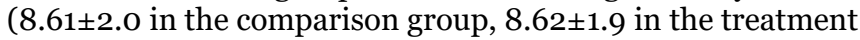
group). Two months after the onset of the therapy, the overall MGPI significantly decreased in each patient group. The total NSPD was $4.47 \pm 1.5$ in the comparison group versus $4.64 \pm 1.7$ in the treatment group. NSPDs in both sensory and affective scales were approximately equal, and almost halved as a result of treatment, while MGPI decreased more in the treatment group. The affective-emotional component of the MGPI diminished by 2.7 times in the comparison group, and by 2.5 times in the treatment group.

When assessing the baseline data according to BDI, patients of both clinical groups revealed the presence of depressive symptoms. The average index values in the groups were within 15 points, which was interpreted as mild (subdepression) or moderate depression (Figure 3). Repeated testing two months later revealed a significant reduction in the severity of depressive symptoms in both groups. The average total score on the Beck scale was about 10 points, which could no longer be considered as the presence of depression. It should be emphasized that in the comparison group, the decrease in this indicator was slightly more substantial than in the treatment group of patients.

Analysis of the baseline STAI data in clinical groups presented the predominance of trait anxiety over reactive anxiety (Figure 4). Average values of reactive (or situational) anxiety corresponded to its moderate manifestations, whereas trait anxiety values matched high anxiety level. The conducted therapy significantly influenced reactive anxiety (there was a reduction, on average, by 10 points), while trait anxiety, being a constitutional feature, declined, on average, just by 5 points.

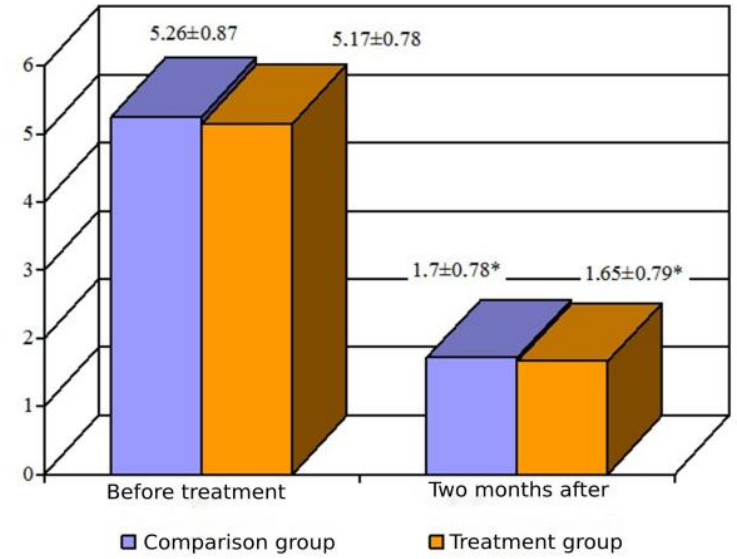

Figure 1. VAS scores of headache intensity in patient groups before and two months after the onset of treatment (M $\pm \mathbf{S D})$. Statistical significance of differences, $\mathrm{p} \leq \mathrm{0.05}$

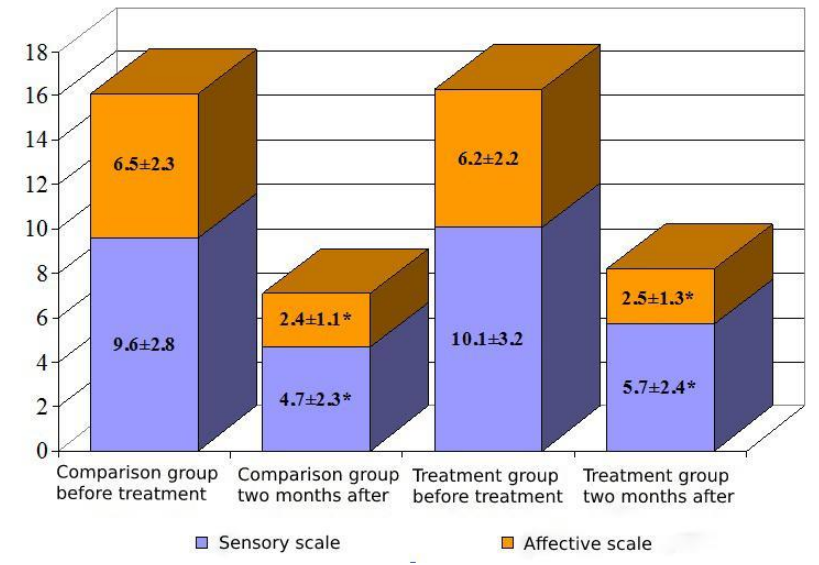

Figure 2. Pain rank index in patient groups before and two months after the onset of treatment according to McGill Pain Questionnaire (M \pm SD)

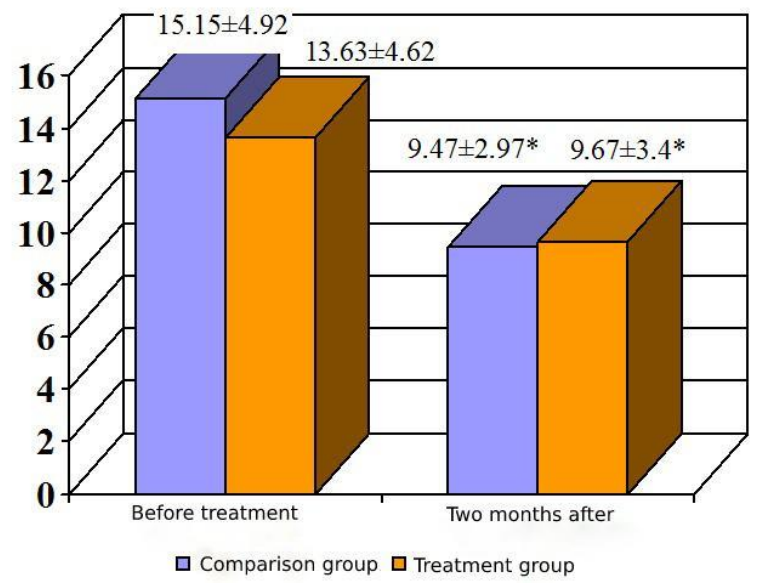

Figure 3. Indicators of depression severity (according to BDI) in patient groups before and two months after the onset of treatment $(M \pm S D)$

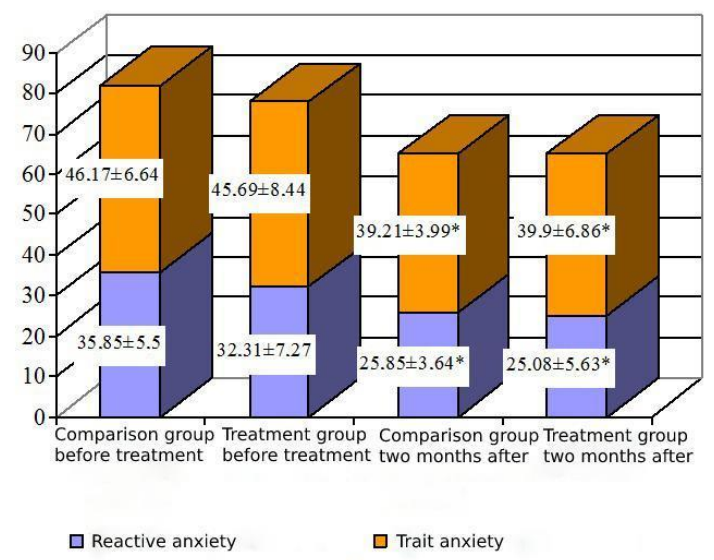

Figure 4. Indicators of reactive vs. trait anxiety according to STAI sensu Ch. Spielberger and Yu. Khanin. in patient groups before and two months after the onset of treatment $(\mathrm{M} \pm \mathbf{S D})$ 


\section{Discussion}

Despite different treatment approaches, the results obtained in the clinical groups of patients were comparable. There was a significant decrease in the headache intensity in both groups of patients.

According to McGill Pain Questionnaire, there was a discrepancy in the interpretation of pain on the VAS scale. This discrepancy was accounted for by the emotional lability of patients, duration of the disease, as well as by low efficacy of used analgesics. However, two months after the onset of treatment, the mean values on the evaluative scale corresponded to the weak pain intensity and did not contradict the interpretation of the results on the VAS scale.

An increase in the MGPI values sensu McGill Pain Questionnaire indicated the prevailing nociceptive component of pain, mainly due to the indicators of the sensory scale. The sensory scale reflects the somatic perception of pain and shows the significance of peripheral sensitization (as one of the links of CTTH pathogenesis) in the formation of a pain syndrome. Patients were choosing the descriptors of the sensory-discriminative group, characterizing pain in terms of mechanical action: 'squeezing', 'pressing', 'suffocating', etc. The presence of central sensitization (i.e., an involvement of the reticular formation and limbic structures in the formation of pain syndrome) was indicated by selected descriptors of the motivational-affective group: 'troublesome', 'exhausting', 'annoying', 'miserable', etc. Number of selected pain descriptors (NSPD), used by patients to describe the nature of pain in the affective and sensory scales, were approximately the same. The results obtained after two months of treatment may have reflected an improvement in the emotional background and mood of patients, and a positive subjective assessment of the treatment. A reduction in MGPI values according to sensory and affective scales indicated the effect of the applied treatment techniques on both peripheral and central components of pain.

Long-term pain syndrome limits the social, professional, and personal capabilities of a person. The emotional-affective component of pain is closely related to various anxietydepressive disorders. In our study, a significant decrease in the frequency and severity of pain episodes had a positive effect on the mental health of patients, which was reflected in reduction of depressive symptoms sensu BDI in both clinical groups.

Analysis of the results obtained using the SpielbergerKhanin questionnaire showed an increase in the level of reactive and trait anxiety in patients, compared with normal values in the population. It is worth noting that high values of trait anxiety suggest the development of an anxiety feeling in CTTH patients in various life situations, including their reaction to pain syndrome, which additionally contributes to the chronicity of pain. After two months of treatment in clinical groups, the values of reactive anxiety decreased and were interpreted as a low level, which implied a diminution in the effect of a provoking stress factor (pain syndrome) on its development.

The effectiveness of applying the pharmacopuncture technique can be explained by its multilevel effect on the CTTH pathogenesis. These acupuncture points are in the area of responsibility of the trigeminovascular and trigeminocervical complexes, where microinjections of the anesthetic cause blockade of the point (mesotherapy) with interruption of nociceptive sensitivity for 20-30 minutes. At the same time, this eliminates the provocation of a headache attack immediately after the procedure and enables the use of this technique during an attack. The change in the tissue volumetric characteristics in response to the injection, its microtrauma, as well as the effect of the decay products of the anesthetic (moderate myotoxicity, vasodilation) create a prolonged stimulation of the biologically active point. This provides a local, segmental and general reflexological response: an impact on the peripheral and central components of the pain syndrome.

\section{Conclusion}

The results of our study suggest the possibility of a variable approach to treating the CTTH patients, in which reflexology techniques may serve a strong addition, or else be an alternative to medicamentous treatment.

The use of pharmacopuncture in CTTH patients can significantly reduce the headache intensity and diminish pharmaceutical drug load, thereby minimizing the risk of side effects. Also, it has a positive effect on concomitant anxietydepressive disorders.

\section{Conflict of interest}

The authors declare no conflicts of interest pertaining to the study.

\section{References}

1. Ghadiri-Sani M, Silver N. Headache (chronic tension-type). BMJ Clin Evid 2016; 1205

https://www.ncbi.nlm.nih.gov/pmc/articles/PMC4747324/

2. Bendtsen L, Evers S, Linde M, et al. EFNS guideline on the treatment of tension-type headache: Report of an EFNS task force. Eur J Neurol 2010; 17(11): 1318-25. https://doi.org/j.14681331.2010.03070.x

3. Akhmadeyeva LR, Azimova YuE, Karakulova YuV, et al. Clinical guidelines on the treatment and diagnostics of the tension-type headache. Russian Medical Journal 2016; 24 (7): 411-9. [In Russian].

4. Linde K, Allais G, Brinkhaus B. Acupuncture for the prevention of tension-type headache. Cochrane Database Syst Rev 2016; 4: CDoo7587.

https://doi.org/10.1002/14651858.CDoo7587.pub2.

5. Sun Y, Gan TJ. Acupuncture for the management of chronic headache: A systematic review. Anesth Analg 2008; 107 (6): 2038-47. https://doi.org/10.1213/ane.obo13e318187c76a

6. Jiang $\mathrm{H}$, Zhang $\mathrm{X}, \mathrm{Lu} \mathrm{J}$, et al. Antidepressant-like effects of acupuncture-insights from DNA methylation and histone modifications of brain-derived neurotrophic factor. Front Psychiatry 2018; 9: 102.

https://doi.org/10.3389/fpsyt.2018.00102.

7. Medvedeva LA, Zagorulko OI, Shevtsova GE. Reflexology of chronic pain: Clinical practice from the standpoint of evidencebased medicine. Russian Journal of Pain 2017; 53 (2): 74-81. [In Russian]. 
8. Koreshkina MI. Current approaches to the tension-type headache treatment. Pharmateca 2013; 272 (19): 22-5. [In Russian].

9. The International Classification of Headache Disorders, 3rd edition (beta version). Cephalalgia 2013; 33 (9): 629-808. https://doi.org/10.1177/0333102413485658.

10. A standard international acupuncture nomenclature:

Memorandum from a WHO meeting. Bull World Health Organ 1990; 68 (2): 165-9. https://pubmed.ncbi.nlm.nih.gov/2364475

Authors:

Lyudmila N. Belimova - Graduate Student, Department of Nervous Diseases and Neurosurgery, Rostov State Medical University, Rostov, Russia;

Victor A. Balyazin - DSc, Professor, Chair of the Department of Nervous Diseases and Neurosurgery, Rostov State Medical University, Rostov, Russia. 